\title{
Manifestaciones Orales en Pacientes Reumatológicos: una Revisión de los Conocimientos
}

\author{
Oral Manifestations of Rheumatologic Patients: A Knowledge Review
}

Thayane Rafaella Chaar Reis*; Brenna Magdalena Lima Nogueira*; Melissa Cristina Lantigua Domínguez*; Sílvio Augusto Fernandes de Menezes $^{* \star *}$; Patrícia de Almeida Rodrigues da Silva e Souza* \& Tatiany Oliveira de Alencar Menezes ${ }^{* \star *}$

REIS, T. R. C.; NOGUEIRA, B. M. L.; DOMÍNGUEZ, M. C. L.; DE MENEZES, S. A. F.; DA SILVA E SOUZA, P. A. R. \& MENEZES, T. O. A. Manifestaciones orales en pacientes reumatológicos: una revisión de los conocimientos. Int. J. Odontostomat., 9(3):413-418, 2015.

RESUMEN: La cavidad oral a través de sus estructuras, dientes y mucosas, desempeñan diversas funciones orgánicas. Actúan en el proceso masticatorio, en la percepción de los sabores, además de servir de vía respiratoria. La relación entre alteraciones sistémicas y manifestaciones orales está bien relatada en la literatura. Observándose en numerosos estudios, palabras claves de morbilidades primariamente bucales, seguidas del diagnóstico de trastornos sistémicos. Las enfermedades reumáticas pueden manifestarse con alteraciones de la condición bucal, además de las presentaciones clínicas peculiares a cada morbilidad. Para la realización de este trabajo fueron investigados en la literatura estudios que evidencien la presencia de manifestaciones orales en enfermedades reumáticas a través de la búsqueda en las bases de datos Lilacs, PubMed y Bireme, incluidos en el año 2009-2015. Las evidencias existentes en la literatura nos revelan la importancia de la inclusión de la evaluación clínica de la boca y sus estructuras anexas, como elemento importante en el diagnóstico auxiliar y orientación para el manejo adecuado de las quejas de estos pacientes.

PALABRAS CLAVE: manifestaciones orales, lupus eritematoso sistemático, enfermedades reumáticas, artritis reumatoide, síndrome de Sjögren.

\section{INTRODUCCIÓN}

La cavidad oral presenta diversas funciones (Martins et al., 2013), con una interacción y equilibrio entre todas las estructuras orales. La mucosa oral presenta características morfofisiológicas diferentes, dependiendo de su localización. Las glándulas salivales y los dientes, cuando se alteran, ocasionan modificaciones en las funciones. Así, el diagnóstico de una serie de desórdenes orgánicos se encuentra íntimamente asociado con manifestaciones orales (Cope \& Cope, 2011).

El estado de la salud oral proporciona evidencias sobre el comportamiento sistémico del paciente. Los tejidos orales están en un directo contacto físico con el cuerpo, relacionándose a través de la sangre, sistema linfático y nervioso. Influencias sistémicas, inmunológicas y psicológicas están en equilibrio con la salud oral y estado general del individuo, por lo que manifestaciones orales pueden surgir como primeras señales de un desorden sistémico (Scarabelot et al., 2011).

Alteraciones como el lupus eritematoso sistémico, síndrome de Sjögren, esclerosis sistémica, dermatomiositis, enfermedad de Behcet y artritis reumatoide, son las enfermedades reumatológicas que comúnmente presentan manifestaciones en la cavidad oral. Entre los signos más frecuentes se destacan: ulceraciones orales, xerostomía, procesos cariosos en locales raros y lesiones mucosas con características peculiares (Cope \& Cope); y aun se citan la microstomía, reabsorción alveolar y edema glandu-

\footnotetext{
*Estudiante de Odontología, Escuela de Odontología de la Universidad Federal de Pará, Belém, Brasil.

**Departamento de Endodoncia, Escuela de Odontología de la Universidad Federal de Pará, Belém, Brasil.

***Departamento de Periodontología, Centro Universitário de Pará, Belém, Brasil.

****Departamento de Pacientes con Necesidades Especiales, Escuela de Odontología de la Universidad Federal de Pará, Belém, Brasil.
} 
lar (Scarabelot et al.). El objetivo de esta revisión fue describir la presencia de manifestaciones orales asociadas a desórdenes reumáticos y otras alteraciones características de la cavidad oral.

\section{MATERIAL Y MÉTODO}

Los artículos fueron seleccionados a partir Lilacs, PubMed y bases de datos Bireme, incluidos en el año 2009-2015. Se utilizaron las siguientes palabras clave: Manifestaciones orales, lupus eritematoso sistémico, enfermedades reumáticas, la artritis reumatoide y síndrome de Sjögren.

\section{RESULTADOS Y DISCUSIÓN}

En enero del 2000, la Organización Mundial de la Salud declaró la década 2000-2010 como la "Década de los huesos y las articulaciones". En gran parte debido a las enfermedades reumáticas, alcanzando casi el $32 \%$ de la población y predilección por el sexo femenino. El aumento de la incidencia de estas enfermedades musculoesqueléticos se describe como preocupante en razón al alto costo que estas enfermedades generan a los sistemas de salud (Carvalho et al., 2009).

La interacción entre la odontología y las enfermedades reumáticas ha sido destacada por algunos autores. Se ha demostrado que la osteoporosis es capaz de reducir la densidad del hueso de la mandíbula, lo que puede causar fracturas óseas durante las extracciones. Por otra parte, los pacientes reumáticos pueden tener progresión acelerada de enfermedades periodontales. El término "enfermedades reumáticas" es muy amplio e engloba alrededor de 120 enfermedades, siendo la artritis reumatoide una de las principales. Se estima que en Brasil más de $1 \%$ de la población (aproximadamente un millón de personas) es portadora de la enfermedad (Carvalho et al.).

Lupus eritematoso sistémico. El lupus eritematoso sistémico (LES) es una enfermedad autoinmune multisistémica crónica, cuya característica más llamativa, desde el punto de vista clínico y patológico, es el desarrollo de focos inflamatorios en varios tejidos y órganos del cuerpo, como por ejemplo: piel, articulaciones, riñones, vasos sanguíneos, pulmones y corazón. Se trata de una enfermedad rara que causa morbilidad y mortalidad significativas debido a su actividad inflamatoria y procesos infecciosos secundarios a la inmunosupresión inducida por la enfermedad y su tratamiento, así como las complicaciones cardiovasculares (de Araújo et al., 2015).

Los factores ambientales y hormonales en consonancia con la predisposición genética conducen a alteraciones inmunológicas, con excesiva producción de anticuerpos y formación de complejos antígenoanticuerpo. El cuadro clínico varía desde manifestaciones agudas o insidiosas, leves, graves, intermitentes y persistentes. Los síntomas aparecen combinados y no de manera específica (Delfino et al., 2014).

Las mujeres se ven afectadas con más frecuencia que los hombres, al igual que las personas de ascendencia asiática. La edad típica de aparición es entre 20 y 40 años, sin embargo, el LES puede aparecer en la infancia. La incidencia anual estimada de LES en la infancia es 0,6/100.000. La prevalencia mundial oscila entre 12 y 50 por 100.000 , dependiendo de la ubicación y el origen étnico (Leite et al., 2015).

Para el diagnóstico del LES se toman en cuenta criterios clínicos, que se manifiestan en el tiempo, y laboratoriales. El American College of Rheumatology establece once en el diagnóstico de LES, en lo cual el paciente debe presentar al menos cuatro para ser considerado enfermo, que son: eritema malar, lesión discoide, fotosensibilidad, úlceras nasales/orales, serositis, artritis, insuficiencia renal, alteraciones neurológicas, alteraciones hematológicas, alteraciones inmunológicas y los anticuerpos antinucleares (Assis \& Baaklini, 2009).

Las manifestaciones bucales han sido reportadas en 20 a $45 \%$ de los pacientes con LES, con un aumento de las lesiones generalmente ocurriendo durante las fases activas de la enfermedad. Los sitios preferidos de las lesiones bucales, con el fin de disminuir la prevalencia, es la mucosa bucal, encías y mucosa labial. Por otra parte, los fármacos utilizados para el tratamiento de LES pueden resultar con alteraciones en la mucosa, tornando difícil la caracterización de la etiología de lesiones orales en estos pacientes (Dghoughi et al., 2010).

El pronóstico de los pacientes con la enfermedad con compromiso sistémico depende cuales ór- 
ganos fueron afectados y de la frecuencia de reactivación de la enfermedad. De manera general, para los pacientes que están recibiendo tratamiento hoy en día, la tasa de supervivencia en cinco años es de 82 a $90 \%$, pero esta cifra cae para $63-75 \%$ en 20 años (Assis \& Baaklini).

Síndrome de Sjögren. El síndrome de Sjögren primario (SSp) es una enfermedad sistémica caracterizada por la infiltración linfocítica y la destrucción progresiva de las glándulas exocrinas. Sin embargo, el proceso inflamatorio puede afectar cualquier órgano. Las manifestaciones clínicas pueden ser divididos en dos aspectos: (1) benigna, pero debilitantes, tales como sequedad, dolor y fatiga, que afecta a la mayoría de los pacientes y (2) sistémica, que puede ser potencialmente grave y afecta a $20-40 \%$ pacientes. La prevalencia puede variar de $0,03 \%$ a $2,7 \%$ de la población mundial (Miyamoto et al., 2015).

Puede ocurrir en todos los grupos de edad y es rara en niños. Afecta principalmente a mujeres durante la quinta y sexta década de vida. Sin embargo no hay sustancia química conocida o factores ambientales que estén implicados en la patogénesis del síndrome de Sjögren, se ve con más frecuencia en los pacientes que tienen sensibilidad al sol y en climas secos (Sardenberg et al., 2010).

Dentro de los síntomas orales, se destaca la xerostomía como principal síntoma; lengua fisurada; atrofia papilar; mucosa oral eritematosa y sensible; queilitis angular $y, 1 / 3$ a la mitad de los pacientes, una tumefacción difusa y firme de las glándulas salivales que aumenta durante el curso de la enfermedad. Un síntoma extra-oral bastante característico es la queratoconjuntivitis. Considerando que uno de los principales síntomas del síndrome de Sjögren consiste en la xerostomía, sabiéndose la importancia de las propiedadesinmunológicas de la secreción salival en el mantenimiento del equilibrio del medio oral, sobre todo en la desmineralizaciónremineralización del esmalte dental, durante los momentos de desafíos cariogénicos es que se hace necesario investigar las secuelas de este síndrome (de Sena et al., 2006).

También existe la hipótesis de que las enfermedades periodontales y los cambios en la mucosa oral pueden ser provocadas por la falta o incluso ausencia de elementos de defensa importantes en el contenido de la saliva oral. El flujo salival disminuido contribuye al aumento de la proporción de S. mutans, lactobacilos y cándida. Manchas eritematosas son comunes en la cavidad oral y se asocian con el sobrecrecimiento de cándida albicans (Seine et al.).

Esclerosis Sistémica. De etiología desconocida posiblemente desencadenada por factores ambientales en la predisposición genética de un individuo. La ES se caracteriza principalmente por microvasculopatía, la activación de fibroblastos y la producción de colágeno excesiva. La ES se trata de una patología única al exibir carcateristicas de tres procesos fisiopatológicos distintos: consiste en la tríada de lesión vascular, la autoinmunidad (humoral y celular) y la fibrosis del tejido y lleva a la participación de la piel, y en varios órganos internos como el pulmón, el corazón y el tracto gastrointestinal, así como los síntomas musculoesqueléticos (Horimoto da Costa, 2015).

Es una enfermedad rara, su incidencia se estima en 3,8-13,9 casos nuevos/1.000.000/ año. Algunos estudios sugieren un aumento de esta enfermedad en los últimos años. Afecta principalmente a mujeres (relación hombre mujer de 3-9/1) entre 30 y 60 años, con un pico de incidencia entre los 40 y los 50 años. La patogénesis es cuestionable, pero hay tres líneas principales de su fisiopatología: hiperactividad de fibroblastos, microangiopatía endoproliferativa y trastornos inmunes sugestivos de autoinmunidad (Ludici et al., 2013).

Los hallazgos orales incluyen microstomía, que se desarrolla debido a la deposición de colágeno en los tejidos periorales, causando la restricción de la apertura oral, formación de arrugas en los surcos periorales, y en las radiografías interproximales y periapicales se observa, agrandamiento difuso del espacio del ligamento periodontal. Como parte de los síntomas de la enfermedad también se ha reportado la presencia de la hipofunción de las glándulas salivales con el consiguiente aumento en la frecuencia de la caries dental y la enfermedad periodontal (Pereira et al., 2009).

Otros hallazgos incluyen xerostomía y la pérdida de inserción de la mucosa gingival con múltiples áreas de retracción. Los pacientes con esclerodermia tienen una mayor prevalencia de enfermedad periodontal en comparación con los individuos sin la enfermedad. La microvasculopatía obliterante es una característica de la esclerosis sistémica. Se puede encontrar la acumulación de placa debido a la limita- 
ción de la apertura de cavidad oral y la dificultad del manejo del cepillo dental causado por los cambios escleróticos en los dedos y las manos.

Se recomienda la terapia de flúor cada tres meses. Procedimientos de ortodoncia se pueden realizar para estabilizar los dientes con pérdida de soporte óseo (Dghoughi et al.).

Dermatomiositis. Dermatomiositis (DM) es una enfermedad multisistémica de probable etiología autoinmune, que se caracteriza por el desarrollo de una inflamación no supurativa en el músculo esquelético. También como posibles síntomas generales y hallazgos típicos, se puede observar la participación cardiopulmonar y gastrointestinal, aunque estos casos potencialmente mortales (por ejemplo, el riesgo de evolución para fibrosis pulmonar y, más frecuentemente en pediatría con riesgo de perforación intestinal). En su etiopatogenia se dice que participan en los procesos autoinmunes desencadenadas por factores ambientales en un individuo genéticamente predispuestos (Moreira et al., 2011).

Se estima que la tasa de prevalencia de la dermatomiositis es cerca de uno por cada 100.000 en la población general, predominantemente en las mujeres en una proporción de 2:1. Tiene dos picos de incidencia: 5-14 años (DJM) y 45 a 65, la edad más común de diagnóstico es de 40 años. La debilidad muscular es la señal más comúnmente observada en las primeras etapas. Generalmente se desarrolla gradualmente durante semanas o meses (Di Giácomo et al., 2010).

Las manifestaciones cutáneas se agregan o preceden a las manifestaciones musculares. Entre los principales cambios se encuentran las pápulas Gottron conocidas como formaciones escamosas, elevadas, de color violeta, simétricamente distribuidas, que se encuentra en los nudillos; el heliotropo, erupción característica en el párpado superior; erupción eritematosa y hemorragias de las cutículas (Hak et al., 2011).

Rara vez la dermatomiositis implica tejidos orales como parte del proceso de la enfermedad. En pocos pacientes se observan placas hiperqueratósicas en la mucosa oral similares a las observadas clínicamente en pacientes con lupus eritematoso. La presencia de eritema y manchas blancas en la lengua, el paladar, la mucosa oral y ulceración labial fueron los primeros informes del envolvimiento de la dermatomiositis. La telangiectasia gingival también fue mencionada en la literatura como un síntoma de la dermatomiositis en la infancia y la edad adulta. También puede ser una mayor prevalencia de la caries dental, la acumulación de placa dental y la inflamación gingival y por lo tanto un mayor riesgo de pérdida prematura de dientes (Di Giácomo et al.).

Enfermedad de Behcet. La enfermedad de Behcet (EB) es una alteración inflamatoria multisistémica de etiología desconocida. Clínicamente se caracteriza por úlceras orales y genitales, uveítis, artritis y lesiones cutáneas recurrentes tales como eritema nudoso o pseudofoliculitis. También puede presentar manifestaciones gastrointestinales, pulmonares, neurológicas y cardiovasculares en los casos graves (Madureira et al., 2015).

Hay alrededor de 250 descripciones que pueden haber sido la primera hecha de EB, cuando Hipócrates de Cos relató en su Epidemion, libro 3, caso 7, una enfermedad endémica de en Asia Menor, caracterizada por úlceras aftosas. Aunque el primero en caracterizar una entidad distinta, con sus tres manifestaciones cardinales: úlceras orales aftosas, úlceras genitales y uveítis, fue el dermatólogo Hulusi Behcet la turca (Neto et al., 2012).

La relación de la enfermedad ha sido discutida con la infección precipitante (herpes I y estreptococos). EI EB se encuentra en pacientes que van desde las edades de 15 y 45, con edad promedio de 30 años. Afectando ambos sexos siendo la frecuencia más alta y de peor pronóstico se produce en los hombres. Oriente Medio y Japón aparecen como sitios de incidencia más altas (Pires et al., 2010).

Históricamente, esta enfermedad se caracterizaba por períodos de remisión y exacerbación de sus manifestaciones. Comúnmente observadas, úlceras orales, que suelen ser el primer signo de la enfermedad, y están presentes hasta el $86,5 \%$ de los adultos y niños. Puede ser recurrente o de forma aislada. Presente aspecto similar a las formas leves y graves de la estomatitis aftosarecurrente y la cicatriz puede ocurrir ocasionalmente cuando se asocia con la forma severa. Sintomatología dolorosa, que mide de 1 a 3 $\mathrm{cm}$ de diámetro, de base amarillenta (presencia de fibrina) y halo eritematoso, simple o múltiple. Puede afectar a cualquier zona de la mucosa oral, a pesar de que son comunes en el velo del paladar y la faringe. Por lo general, una duración de 7 a 14 días y desaparece sin dejar cicatriz (Pires et al.). 
Artritis reumatoide. La artritis reumatoide es un término inespecífico que significa "inflamación de las articulaciones". Esta enfermedad constituye la principal alteración articular degenerativa y se considera una etiología autoinmune de la enfermedad, todavía no está esclarecido. Puede afectar codos, hombros, cuello, caderas, rodillas, tobillos, pies y articulaciones temporomandibulares, debido a la erosión de los huesos y cartílagos que puede involucrar grandes y pequeñas articulaciones, que aparecen junto con manifestaciones sistémicas. Cepillarse los dientes es para la mayoría, una tarea dolorosa, por lo que la salud oral del paciente por lo general es renegada (García et al., 2012).

Se produce a nivel mundial y se estima que 0,3 a $1 \%$ de la población se ve afectada por la enfermedad. Aproximadamente el $80 \%$ de los casos de pacientes con AR en desarrollo están en la edad de 35 a 50 años, y su prevalencia aumenta con la edad (Costa et al., 2015).

Hoy en día se ha incrementado el número de estudios de evaluación de la existencia de una asociación entre la periodontitis y AR en el que se sugiere que los pacientes con AR tienen un mayor riesgo de desarrollar periodontitis, tienen una alta pérdida de dientes en comparación con individuos sanos. Aunque actualmente no hay explicación clara para justificar la simultaneidad entre la enfermedad periodontal y la artritis reumatoide (de Pablo et al., 2009).

La asociación entre la periodontitis y AR se muestra significativa. Esta relación se explica por el hecho de que la liberación continua de productos bacterianos subgingivales resultados en la producción de altos niveles de citoquinas pro-inflamatorias que alcanzan el torrente sanguíneo, proporcionan la aparición de complicaciones sistémicas inflamatorias tales como AR. Además, se sabe que Porphyromonas gingivalis $(\mathrm{Pg})$, c periodontopatógeno fundamental, puede estar asociado con la causa o perpetuación del cuadro clínico de AR, ya que esta es la única procariota capaz de expresar la enzima peptidilarginina deiminasa (PAD) que, cuando libera en la bolsa periodontal, puede difundir al movimiento articular sinovial (Goes et al., 2014).

En conclusión la presencia y la variedad de manifestaciones orales en pacientes con enfermedades reumáticas se observan claramente en la literatura. Algunas de estas manifestaciones son importantes como impacto en las vidas de los portadores de estas enfermedades. La evaluación clínica correcta y precisa de la cavidad oral en pacientes con sospecha de estar implicados en el carácter autoinmune de morbilidad puede dar lugar a un mejor manejo de las condiciones orales presentados por estos individuos, siendo una conducta integrada entre el odontólogo y el médico, el elemento clave para el éxito del abordaje terapéutico.

REIS, T. R. C.; NOGUEIRA, B. M. L.; DOMÍNGUEZ, M. C. L.; DE MENEZES, S. A. F.; DA SILVA E SOUZA, P. A. R. \& MENEZES, T. O. A. Oral manifestations of rheumatologic patients: A knowledge review. Int. J. Odontostomat., 9(3):413-418, 2015.

ABSTRACT: The oral cavity has several physiological functions through their structures. Such structures act in the chewing process, the perception of flavors, besides serving airway. The relationship between systemic diseases and oral manifestations is well reported in the literature. It is observed in numerous studies, morbidity descriptions primarily mouth followed by a diagnosis of systemic disorders. Rheumatic diseases exemplify that these can manifest with changes in oral health in addition to the peculiar clinical presentations to each morbidity. For this work we investigated in the literature studies that provided evidence of the presence of oral manifestations in rheumatic diseases by searching Lilacs, PubMed and Bireme databases, included in the year 20092015. The available evidence in the literature, reveal the importance of including clinical evaluation of the mouth and its associated structures as an important element for the diagnosis and guidance for the proper handling of complaints of these patients.

KEY WORDS: oral manifestations, systemic lupus erythematosus, rheumatic diseases, rheumatoid arthritis and Sjögran syndrome.

\section{REFERENCIAS BIBLIOGRÁFICAS}

Assis, M. R. \& Baaklini, C. E. Como diagnosticar e tratar lúpus eritematoso sistêmico. Rev. Bras. Med., 66(9):27485, 2009.

Carvalho, R. B.; Teixeira, L. P. \& Gomes, M. J. Autopercepção e condições de saúde bucal nos pacientes assistidos pelo Ambulatório de Reumatologia do Hucam/Ufes, Vitória - ES. RFO, 14(3):216-21, 2009.

Cope, G. \& Cope, A. Diagnosis and treatment of oral medicine conditions. Dent. Nurs., 7(10):560-563. 2011.

Costa, J. O.; Lemos, L. L. P.; Machado, M. A. A.; Almeida, A. M.; Kakehasi, A. M.; Araújo, V. E.; Cherchiglia, M. L.; 
Andrade, E. L. G. \& Acurcio, F. A. Infliximabe, metotrexato e sua combinação no tratamento da artrite reumatoide: revisão sistemática e metanálise. Rev. Bras. Reum., 55(2):146-58, 2015.

de Araújo, A. L. P. K.; Paliares, I. C.; de Araújo, M. I. P. K. A.; Novo, N. F.; Cadaval, R. A. M. \& Martinez, J. E. A associação fibromialgia e lúpus eritematoso sistêmico altera a apresentação e a gravidade de ambas as doenças. Rev. Bras. Reum., 55(1):37-42, 2015.

de Pablo, P.; Chapple, I. L.; Buckley, C. D. \& Dietrich, T. Periodontitis in systemic rheumatic diseases. Nat. Rev. Rheumatol., 5(4):218-24, 2009.

de Sena, M. F.; Junior, J. F. L. \& Ferreira, M. A. F. Condição oral dos pacientes com Síndrome de Sjögren: uma revisão sistemática. Rev. Bras. Promoç. Saúde, 19(4): 234-239, 2006.

Delfino, J. A. S.; Pereira, W. M. G. \& Guimarães, R. B. Lúpus eritematoso sistêmico - Relato de caso. Rev. Interfaces Saúde Hum. Tecnol., 2(6), 2014. DOI: $10.16891 / 2317.434 X .141$

Di Giácomo, C. G.; Bueno, A. N.; Vaz, J. L. P. \& da Fonseca Salgado, M. C. Atualização em dermatomiosite. Rev. Bras. Clin. Med. São Paulo, 8(5):434-9, 2010.

Dghoughi, S.; El Wady, W. \& Taleb, B. Systemic sclerosis. Case report and review of literature. N. Y. State Dent. J., 76(3):30-5, 2010.

Garcia, P. O.; dos Santos, S. P. \& de Mendonça, S. M. S. Manifestações bucais em pacientes portadores de artrite Reumatóide. Pós em Revista, 5:276-82, 2012.

Goes, P.; Coelho, A. C. \& Oliveira, C. C. Efeito da raspagem e alisamento radicular em pacientes com artrite reumatoide: revisão da literatura. Rev. Fac. Odontol. Lins/Unimep, 24(1):33-40, 2014.

Hak, A. E.; de Paepe, B.; de Bleecker, J. L.; Tak, P. P. \& de Visser, M. Dermatomyositis and Polymyositis: New Treatment Targets on the Horizon," Neth. J. Med., 69(10):410-21, 2011.

Horimoto, A. M. C. \& da Costa, I. P. Sobreposição de esclerose sistêmica e artrite reumatoide: uma entidade clínica distinta? Rev. Bras. Reum., 2015. DOI: 10.1016/j.rbr.2014.12.011

Leite, C. A.; Galera, M. F.; Espinosa, M. M.; de Lima, P. R. T.; Fernandes, V.; Borges, Á. H. \& Dias, E. P. Prevalence of hyposalivation in patients with systemic lupus erythematosus in a brazilian subpopulation. Int. J. Rheum., 2015:730285, 2015.
Madureira, P.; Rodrigues, M.; Serrano, E.; Vítor, A. B. \& Brito, I. Trombose intracardíaca na doença de Behçet: evento com risco de vida. Rev. Bras. Reum., 2014. DOI: 10.1016/ j.rbr.2014.11.001

Martins, G. S. B.; Rocha, C.; Esteves, I. M. Z. S.; Maia, J. L.; de Arruda, L. M. T.; Zanatta, L. H. S.; Cesetti, M. V. \& Costa, G. P. Avaliação da prevalência de alterações dermatológicas em pacientes com lúpus eritematoso sistêmico. Rev. Med. Saúde Brasilia, 2(3):125-32, 2013.

Miyamoto, S. T.; Paganotti, M. A.; Serrano, É. V.; Giovelli, R. A. \& Valim, V. Avaliação da fadiga e da secura na síndrome de Sjögren primária: versão brasileira do "Profile of Fatigue and Discomfort - Sicca Symptoms Inventory (short form) (Profad-SSI-SF)". Rev. Bras. Reum., 55(2):113-22, 2015.

Moreira, A.; Pereira, O.; Carvalho, S.; Vieira, M. J.; Salgado, M. \& Fernandes, A. P. Um caso de dermatomiosite juvenil. Acta Peditr. Port., 42(1):20-3, 2011.

Neto, N. B. F.; da Silva Rigo, V.; da Cunha, V. R. \& de Oliveira, F. P. Mielite longitudinal secundária à síndrome de Behçet. Relato de caso. Rev. Bras. Clin. Med. São Paulo, 10(3):256-9, 2012.

Pereira, M. C. M. C.; Nunes, R. A. M.; Marchionni, A. M. \& Martins, G. B. Esclerodermia Sistêmica: relato de caso clínico. Rev. Odont. Univ. Cid. São Paulo, 21(1):69-73, 2009.

Pires, P. J. S.; Martha, V. F.; Mallmann, L. B. \& Steffen, N. Doença de Behçet e a visão do otorrinolaringologista. Rev. AMRIGS, 54(3):334-6, 2010.

Sardenberg, F.; Goursand, D.; Polletto, L. T.; Vale, M. P.; Zarzar, P. M. \& Paiva, S. M. Oral manifestations and treatment of a child with Sjögren's syndrome. J. Dent. Child (Chic.), 77(2):102-5, 2010.

Scarabelot, V. L.; Gutierrez, L. M. O.; Souza, A.; Oliveira, M. G.; Chaves, A. C. M.; Munerato, M. C.; Hidalgo, M. P.; Caumo, W. \& Torres, I. L. Avaliação de fatores associados ao diagnóstico de queimação bucal: um estudo preliminar. Rev. HCPA, 31(3):318-25, 2011.

Dirección para Correspondencia:

Brenna Magdalena Lima Nogueira

Departamento de Endodoncia

Escuela de Odontología

Universidad Federal de Pará

Belém, Pará

BRASIL

Email: bre_nog@hotmail.com

Recibido : 30-06-2015

Aceptado: 28-10-2015 